Research Article

\title{
Development of Dimethylaminoethyl Methacrylate Based Nanoparticulate Drug Delivery System Using Nanoprecipitation Method and Optimization of Process Parameters Using Plackett-Burman Factorial Design
}

\author{
Moorthi Chidambaram $~$, Kathiresan Krishnasamy \\ Department of Pharmacy, Annamalai University, Annamalai Nagar - 608 002, Chidambaram, Tamil Nadu, India \\ $\sim$ Corresponding author: E-mail: cmoorthitgodu@gmail.com
}

Received: Oct. 31, 2013; Accepted: Jan. 5, 2014; Published: March 12, 2014.

Citation: Moorthi Chidambaram and Kathiresan Krishnasamy. Development of Dimethylaminoethyl Methacrylate Based Nanoparticulate Drug Delivery System Using Nanoprecipitation Method and Optimization of Process Parameters Using Plackett-Burman Factorial Design. Nano Biomed. Eng. 20I4, 6(I), 19-30.

DOI: $10.5101 /$ nbe.v6il.p19-30.

\begin{abstract}
The present study was aimed to develop dimethylaminoethyl methacrylate based nanoparticulate drug delivery system using nanoprecipitation method and optimize the process parameters using PlackettBurman factorial design to yield least average particle size and narrow sized particle distribution without filtration or centrifugation process. Twelve experimental runs involving 11 process parameters at higher and lower levels were generated using Design-Expert. Factorial design result has shown that (a) Except stirring duration all other process parameters significantly influence the average particle size; (B) Except $\beta$-cyclodextrin concentration, aqueous phase volume and organic phase volume, all other process parameters significantly influence the polydispersity index; and (C) Except polymer concentration and poloxamer 407 concentration, all other process parameters do not significantly influence the zeta potential. The average particle size, polydispersity index and zeta potential of the prepared dual drug loaded nanoparticles were well within acceptable limits and found to be in the range of 47 to $87 \mathrm{~nm}, 0.14$ to 0.28 and 22 to $39 \mathrm{mV}$, respectively. Surface morphology examination has shown that the prepared nanoparticles were spherical in shape. The developed dimethylaminoethyl methacrylate based nanoparticulate drug delivery system can be routinely used to fabricate narrow sized polymeric nanoparticles without filtration or centrifugation process.
\end{abstract}

Keywords: Dimethylaminoethyl Methacrylate Based Polymer; Nanoparticulate Drug Delivery System; Plackett-Burman Factorial Design; Polymeric Nanoparticles

\section{Introduction}

Engineering material in nanometer range considerably modifies the physicochemical properties of the engineered material, which offer major development in various fields of science including automotive, electronics, textile, military, energy and medicine. Implementation of nanotechnology in medicine provides significant development in diagnosis and treatment of various disorders via 
nanoparticulate drug delivery system. Nanoparticulate drug delivery system includes solid-lipid nanoparticle, metallic nanoparticle, carbon nanotube, nanocrystal, nanosponge, magnetic nanoparticle, albumin nanoparticle, fullerene nanoparticle and polymeric nanoparticle [1-4]. Each nanoparticulate drug delivery system has its own advantages however, polymeric nanoparticles provides sizeable advantages including improvement in aqueous solubility, protection to the encapsulated drug from degradation, enhancement in bioavailability, provides controlled drug release, reduces the drug toxicities and targets the drug to specific site [5-7]. Extensive research work has been reported to fabricate polymeric nanoparticles, which include solvent evaporation, salting-out, nanoprecipitation, dialysis, spray drying, desolvation, supercritical fluid technology and ionic gelation [8-14]. However, nanoprecipitation is the most suitable method to fabricate polymeric nanoparticles but the major disadvantage with nanoprecipitation method is the higher polydispersity index (i.e. particles with broad distribution), which require filtration or centrifugation process to yield particles with narrow distribution [15]. Dimethylaminoethyl methacrylate based polymers are co-polymers synthesized from acrylic and methacrylic acid esters. These polymers are biocompatible and offer cationic charge to the particle surface, which may reduce the particle aggregation resulting in narrow sized distribution. These polymers have been extensively used for taste masking, odor masking and moisture protection of various pharmaceutical preparations. However, the present study was aimed to develop dimethylaminoethyl methacrylate based nanoparticulate drug delivery system using nanoprecipitation method and optimize the process parameters using Plackett-Burman factorial design to yield least average particle size and narrow sized particle distribution without filtration or centrifugation process.

\section{Experimental section}

\section{Material}

Dimethylaminoethyl Methacrylate based polymer was used as polymer in the study and was obtained from Degussa, India. Curcumin (97\%), Piperine (97\%), Quercetin (98\%) and Silibinin ( $\geq 98 \%)$ were used in the study as model drugs and were obtained from Sigma-Aldrich, India. Analytical grade ethanol was used as organic solvent and was obtained from Brampton, Canada. Analytical grade acetone was also used as organic solvent and was obtained from S.D Fine Chemicals, India. $\beta$-cyclodextrin was used as stabilizer in the study and was obtained from Himedia Laboratories, India. Poloxamer 188 and 407 were used as surfactant and were obtained from Sigma-Aldrich, India.

\section{Development of dimethylaminoethyl methacrylate based nanoparticulate drug delivery system}

Dimethylaminoethyl methacrylate based nanoparticulate drug delivery system was developed using nanoprecipitation method [15]. Briefly, dimethylaminoethyl methacrylate based polymer was dissolved in organic solvent, which was transferred at once into the aqueous phase containing poloxamer and $\beta$-cyclodextrin under mechanical stirring (Remi, India). Polymeric nanoparticles were formed spontaneously and turned the aqueous phase slightly milky with bluish opalescence but the stirring process was continued to aid the size reduction and to evaporate the residual solvent present in the nanoformulation. However, nanoparticle parameters such as particle size, polydispersity index and zeta potential depends on the process parameters including polymer concentration, organic solvent, percentage of organic solvent, organic phase volume, poloxamer concentration, $\beta$-cyclodextrin concentration, aqueous phase volume, beaker volume, stirring speed and stirring duration. Hence, PlackettBurman factorial design was used to optimize the process parameters at lower and higher levels (Table 1). Twelve experimental runs involving 11 process parameters at higher and lower levels were generated using Design-Expert ${ }^{\circledR}$ (Version 7.1.5; Stat-Ease, Inc. USA) (Table 2). Prepared polymeric nanoparticles were characterized for average particle size, polydispersity index and zeta potential using Zetasizer (ZEN3600, Malvern).

\section{Fabrication of plain, curcumin-piperine, curcumin-quercetin and curcumin- silibinin loaded dimethylaminoethyl methacrylate based nanoparticles}

Plain, curcumin-piperine, curcumin-quercetin and curcumin-silibinin loaded polymeric nanoparticles were prepared using final optimized formula from Plackett-Burman factorial design (Table 3). About 
Table 1 Optimization process parameters at lower and higher levels

\begin{tabular}{|c|c|c|c|}
\hline \multirow{2}{*}{ S.No. } & \multirow{2}{*}{ Process parameters } & \multicolumn{2}{|c|}{ Levels } \\
\hline & & Lower & Higher \\
\hline 1. & Polymer concentration & $50 \mathrm{mg}$ & $250 \mathrm{mg}$ \\
\hline 2. & Organic solvent & Acetone & Ethanol \\
\hline 3. & Percentage of organic solvent & $60 \%$ & $100 \%$ \\
\hline 4. & Organic phase volume & $10 \mathrm{~mL}$ & $20 \mathrm{~mL}$ \\
\hline 5. & Poloxamer 188 concentration & $50 \mathrm{mg}$ & $125 \mathrm{mg}$ \\
\hline 6. & Poloxamer 407 concentration & $0 \mathrm{mg}$ & $125 \mathrm{mg}$ \\
\hline 7. & $\beta$-cyclodextrin concentration & $50 \mathrm{mg}$ & $250 \mathrm{mg}$ \\
\hline 8. & Aqueous phase volume & $25 \mathrm{~mL}$ & $50 \mathrm{~mL}$ \\
\hline 9. & Beaker volume & $250 \mathrm{~mL}$ & $500 \mathrm{~mL}$ \\
\hline 10. & Stirring speed & $500 \mathrm{rpm}$ & $1000 \mathrm{rpm}$ \\
\hline 11. & Stirring duration & 1 hour & 3 hours \\
\hline
\end{tabular}

Table 2 Plackett-Burman factorial design

\begin{tabular}{|c|c|c|c|c|c|c|c|c|c|c|c|}
\hline Run & $\begin{array}{c}\mathrm{A} \\
(\mathrm{mg})\end{array}$ & $\begin{array}{c}\mathbf{B} \\
(@ / \ddot{\mathbf{e}})\end{array}$ & $\begin{array}{c}\mathrm{C} \\
(\%) \\
\end{array}$ & $\begin{array}{c}D \\
(\mathrm{~mL})\end{array}$ & $\begin{array}{c}\mathbf{E} \\
\mathrm{mg}\end{array}$ & $\begin{array}{c}F \\
(\mathrm{mg})\end{array}$ & $\begin{array}{c}\mathbf{G} \\
(\mathrm{mg})\end{array}$ & $\begin{array}{c}\mathbf{H} \\
(\mathbf{m L})\end{array}$ & $\begin{array}{c}\mathbf{J} \\
(\mathrm{mL})\end{array}$ & $\begin{array}{c}\mathrm{K} \\
(\mathrm{rpm})\end{array}$ & $\begin{array}{c}\mathbf{L} \\
(\mathbf{H r})\end{array}$ \\
\hline PB01 & 50 & (a) & 60 & 20 & 50 & 0 & 50 & 50 & 250 & 1000 & 3 \\
\hline PB02 & 250 & (a) & 100 & 10 & 50 & 125 & 50 & 50 & 500 & 500 & 3 \\
\hline PB03 & 250 & ë & 60 & 20 & 50 & 125 & 250 & 50 & 250 & 500 & 1 \\
\hline PB04 & 50 & (a) & 60 & 10 & 125 & 125 & 250 & 25 & 250 & 500 & 3 \\
\hline PB05 & 50 & ë & 60 & 10 & 125 & 125 & 50 & 50 & 500 & 1000 & 1 \\
\hline PB06 & 250 & (a) & 60 & 10 & 50 & 0 & 250 & 25 & 500 & 1000 & 1 \\
\hline PB07 & 250 & (a) & 100 & 20 & 125 & 125 & 50 & 25 & 250 & 1000 & 1 \\
\hline PB08 & 50 & (a) & 100 & 20 & 125 & 0 & 250 & 50 & 500 & 500 & 1 \\
\hline PB09 & 250 & ë & 100 & 10 & 125 & 0 & 250 & 50 & 250 & 1000 & 3 \\
\hline PB10 & 50 & ë & 100 & 20 & 50 & 125 & 250 & 25 & 500 & 1000 & 3 \\
\hline PB11 & 50 & ë & 100 & 10 & 50 & 0 & 50 & 25 & 250 & 500 & 1 \\
\hline PB12 & 250 & ë & 60 & 20 & 125 & 0 & 50 & 25 & 500 & 500 & 3 \\
\hline
\end{tabular}

@: Acetone; ë: Ethanol; Hr: Hour

Table 3 Fabrication of plain, curcumin-piperine, curcumin-quercetin and curcumin-silibinin loaded dimethylaminoethyl methacrylate based nanoparticles

\begin{tabular}{|c|c|c|c|c|c|c|c|c|c|}
\hline Dual Drug Combinations* & $\begin{array}{c}\mathrm{A} \\
(\mathrm{mg})\end{array}$ & $\begin{array}{c}\text { B } \\
(\%)\end{array}$ & $\begin{array}{c}\mathrm{C} \\
(\mathrm{mL})\end{array}$ & $\begin{array}{c}\mathrm{D} \\
\mathrm{mg}\end{array}$ & $\begin{array}{c}E \\
(\mathrm{mg})\end{array}$ & $\begin{array}{c}\mathbf{F} \\
(\mathrm{mL})\end{array}$ & $\begin{array}{c}G \\
(m L)\end{array}$ & $\begin{array}{c}\mathrm{H} \\
(\mathrm{rpm})\end{array}$ & $\begin{array}{c}I \\
\text { (min) }\end{array}$ \\
\hline Plain & 250 & $60 \%$ & 16 & 97 & 54 & 49 & 500 & 520 & 80 \\
\hline Curcumin-Piperine* & 250 & $60 \%$ & 16 & 97 & 54 & 49 & 500 & 520 & 80 \\
\hline Curcumin-Quercetin* & 250 & $60 \%$ & 16 & 97 & 54 & 49 & 500 & 520 & 80 \\
\hline Curcumin-Silibinin* & 250 & $60 \%$ & 16 & 97 & 54 & 49 & 500 & 520 & 80 \\
\hline
\end{tabular}

Dual Drug Combinations": $25 \mathrm{mg}$ of curcumin and $25 \mathrm{mg}$ of piperine or quercetin or silibinin; A: Polymer; B: Ethanol; C: Organic phase volume; D: Poloxamer 188; E: $\beta$-cyclodextrin; F: Aqueous phase volume; G: Beaker volume; H: Stirring speed; I: Stirring duration.

$250 \mathrm{mg}$ of dimethylaminoethyl methacrylate based polymer with and without $50 \mathrm{mg}$ of various dual drug combinations (Curcumin $25 \mathrm{mg}+$ Piperine $25 \mathrm{mg}$ ); (Curcumin $25 \mathrm{mg}+$ Quercetin $25 \mathrm{mg}$ ); and (Curcumin $25 \mathrm{mg}+$ Silibinin $25 \mathrm{mg}$ ) were dissolved in $16 \mathrm{~mL}$ of $60 \%$ ethanol. The prepared organic phase was transferred at once into $500 \mathrm{~mL}$ beaker containing 49 $\mathrm{mL}$ of distilled water, $97 \mathrm{mg}$ of Poloxamer 188 and 54 $\mathrm{mg}$ of $\beta$-cyclodextrin under mechanical stirring (RQT$124 \mathrm{~A}, \mathrm{Remi})$ at $520 \mathrm{rpm}$. Polymeric nanoparticles were formed spontaneously but the stirring process was continued for 80 minutes to aid the size reduction and to evaporate the residual solvent. Prepared nanoparticles were characterized for average particle size, polydispersity index and zeta potential using Zetasizer (ZEN3600, Malvern Instrument, UK) and particle surface morphology using transmission electron microscopy (Hitachi H-7500).

\section{Results and Discussion}

Development of dimethylaminoethyl methacrylate based nanoparticulate drug delivery system

Dimethylaminoethyl methacrylate based 
nanoparticulate drug delivery system was developed using nanoprecipitation method. In nanoprecipitation method, addition of organic phase into the aqueous phase results in miscibility of organic solvent into distilled water of the aqueous phase leading to increase in the polarity of organic solvent, which in turn decreases the solubility of polymer. However, nucleation of polymer gets initiated when the equilibrium concentration surpasses the solubility threshold of polymer. Stirring process aid the size reduction of polymer at the initial stage but in the later stages, cationic nature of polymer provided cationic charge to the nanoparticle surface and higher number of likely charged nanoparticles repels each other and creates an electrostatic repulsive force and maintains the nanoparticles in Brownian motion, which is expected to overcome the van der walls attractive force arising from induced dipole-dipole interaction between nanoparticles and gravitational force, thereby stabilize the nanoformulation by preventing the aggregation [16-18]. Prepared polymeric nanoparticles were characterized for average particle size, polydispersity index and zeta potential (Fig.1, Fig.2 and Table 4). In spite of its simplicity, nanoprecipitation method involves many processes, which may influence the quality of nanoparticles. Hence, we have implemented Plackett-Burman factorial design to optimize the process parameters. Plackett-Burman is a two level factorial design, which utilizes least number of experimental runs for maximum process parameters. The effect of each process parameter was determined by the following equation $\mathrm{E}_{\mathrm{xi}}=2$ $\left(\Sigma \mathrm{H}_{\mathrm{xi}}-\Sigma \mathrm{L}_{\mathrm{xi}}\right) / \mathrm{N}$, where $\mathrm{E}_{\mathrm{xi}}$ is the concentration effect of particular parameter, $\mathrm{H}_{\mathrm{xi}}$ is the response at the higher level, $\mathrm{L}_{\mathrm{xi}}$ is the response at the lower level and $\mathrm{N}$ is the total number of experimental runs. Positive sign in the model for a response indicates an effect that favours and negative sign indicates an inverse relationship between responses. The linear equation of Plackett-Burman experimental design is expressed as $\mathrm{R}=\mathrm{b}_{0}+\mathrm{b} 1_{\mathrm{X} 1}+\mathrm{b} 2_{\mathrm{X} 2}+\mathrm{b} 3_{\mathrm{X} 3}+\mathrm{b} 4_{\mathrm{X} 4}+\ldots+\mathrm{bn} \mathrm{Xn}_{\mathrm{Xn}}$, where $\mathrm{R}$ is the response, $\mathrm{b}_{0}$ is the constant and $\mathrm{b} 1, \mathrm{~b} 2 \ldots \mathrm{bn}$ are the coefficients of the parameters X1, X2...Xn [6].

\section{Effect of process parameters on the average particle size}

Average particle size of the prepared polymeric nanoparticles decides its performance such as solubility, dissolution, drug release and cellular uptake [6]. Hence, it is essential to fabricate polymeric nanoparticles with least average particle size (i.e. $<100$ $\mathrm{nm}$ ). Analysis of variance has shown that the process parameters has significant effect (Prob> F, 0.0348) on the average particle size (Table 5). All process parameters except stirring duration significantly influence the average particle size (Table 6 and Fig. 3).

Process parameters such as organic solvent, $\beta$-cyclodextrin concentration, organic solvent percentage, stirring speed, polymer concentration and beaker volume has favourable effect on the average particle size whereas, aqueous phase volume, organic phase volume, poloxamer 188 concentration, poloxamer 407 concentration and stirring duration has inverse relationship with the average particle size (Fig. 3). Moreover, the observed average particle size was comparable with predicted value of PlackettBurman factorial design (Table 7). The linear model explaining the effect of process parameters on the average particle size is given as Average particle size $=$ $+155.09+11.21 * \mathrm{~A}+56.19 * \mathrm{~B}+35.20 * \mathrm{C}-28.74 * \mathrm{D}$ $-15.63 * \mathrm{E}-13.92 * \mathrm{~F}+45.77 * \mathrm{G}-37.50 * \mathrm{H}+9.13 * \mathrm{~J}+$ $16.71 * \mathrm{~K}$.

\section{Effect of process parameters on the polydispersity index}

Polydispersity Index (PDI) measures the distribution

Table 4 Characterization of prepared dimethylaminoethyl methacrylate based polymeric nanoparticles

\begin{tabular}{cccc}
\hline Run & Average Particle Size $(\mathbf{n m})$ & Polydispersity Index & Zeta Potential $(\mathbf{m V})$ \\
\hline 1 & 159.0 & 0.910 & 20.8 \\
2 & 125.5 & 0.060 & 23.2 \\
3 & 102.1 & 0.131 & 19.5 \\
4 & 290.5 & 0.641 & 4.96 \\
5 & 66.04 & 0.311 & 17.2 \\
6 & 426.5 & 0.452 & 31.9 \\
7 & 129.7 & 0.187 & 26.9 \\
8 & 136.5 & 0.205 & 23.0 \\
9 & 116.4 & 0.177 & 36.8 \\
10 & 133.2 & 0.334 & 9.76 \\
11 & 78.07 & 0.166 & 23.1 \\
\hline
\end{tabular}



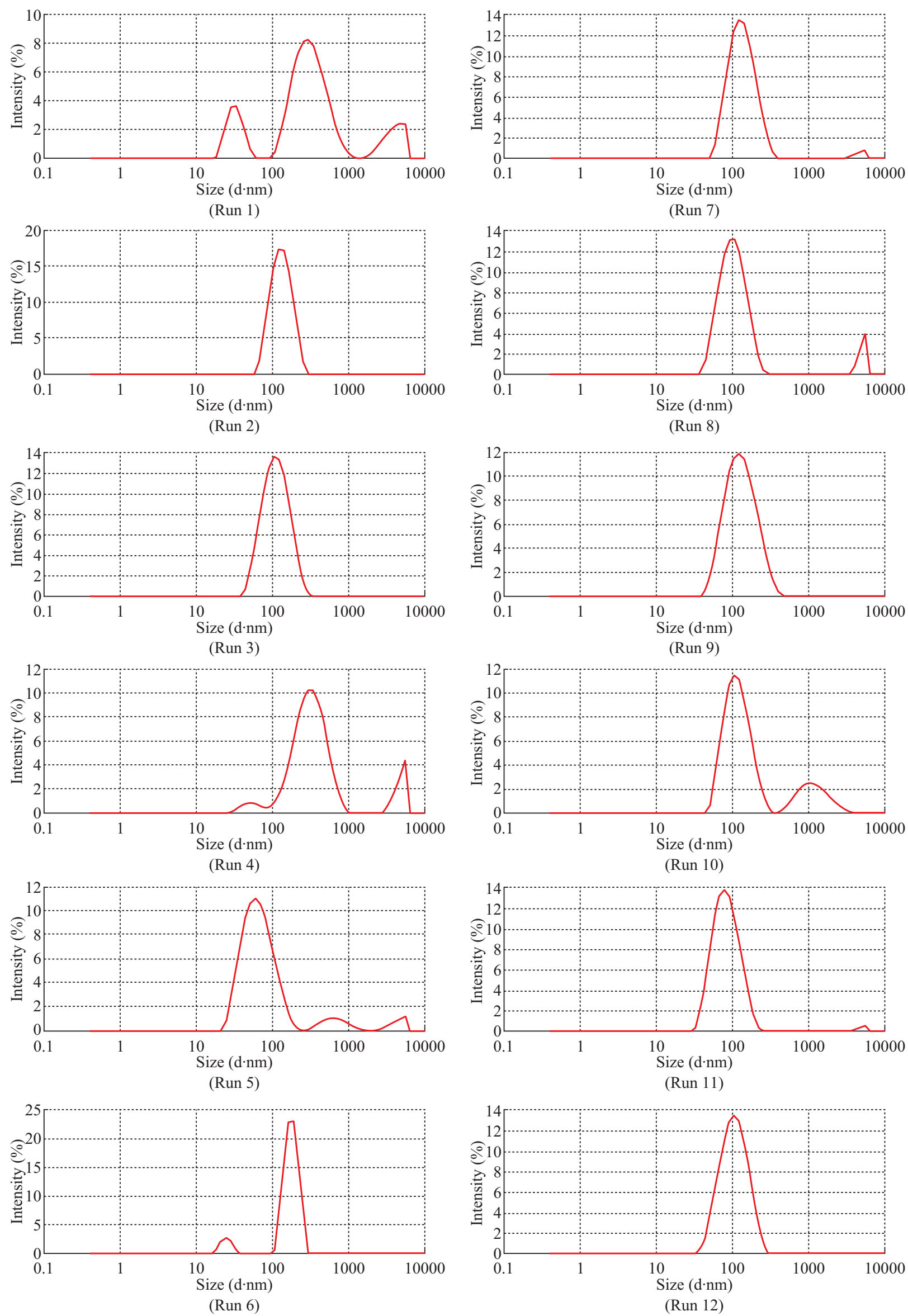

Fig. 1 Particle size spectrum of Run 1-12. 

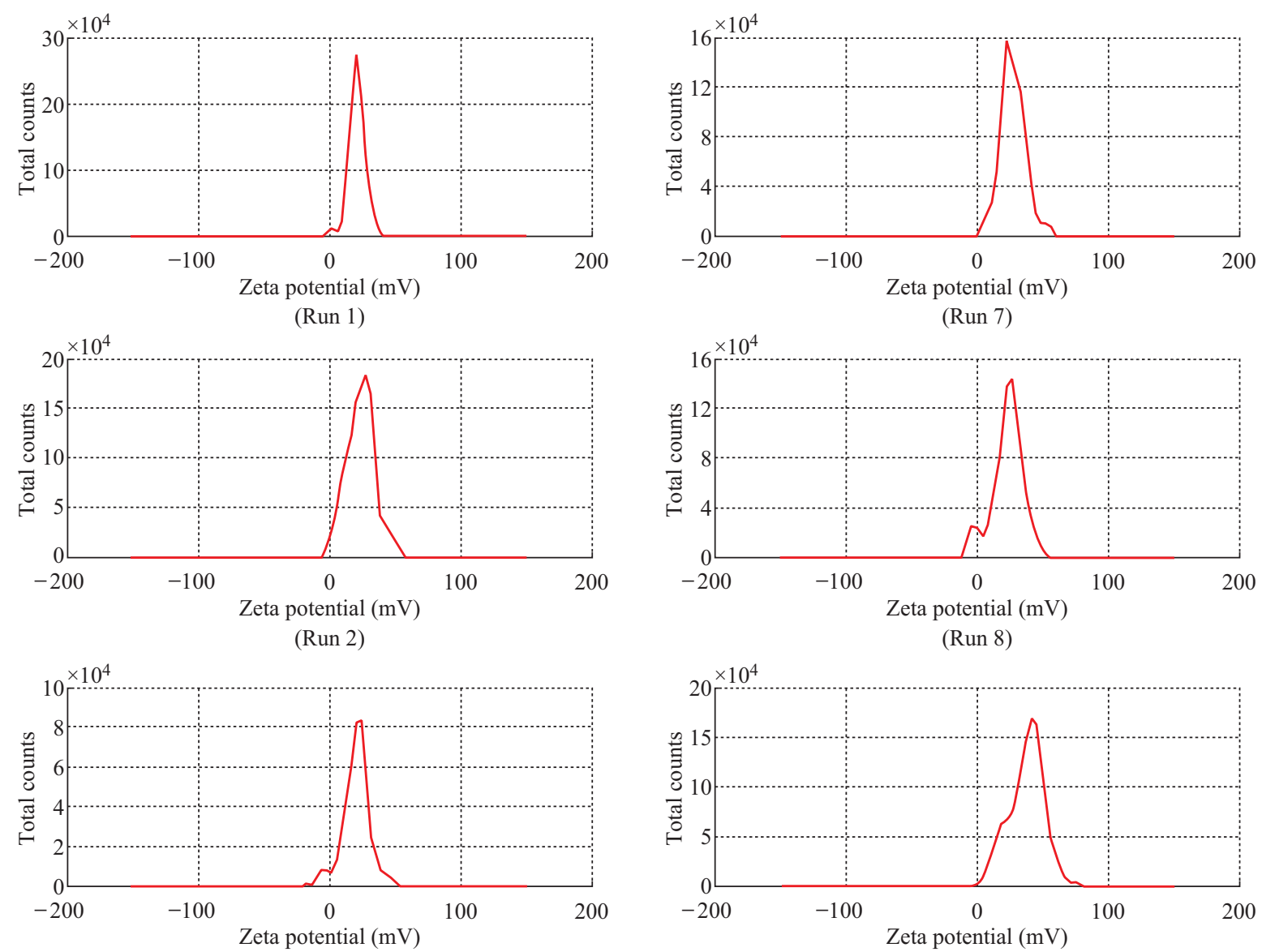

(Run 8)

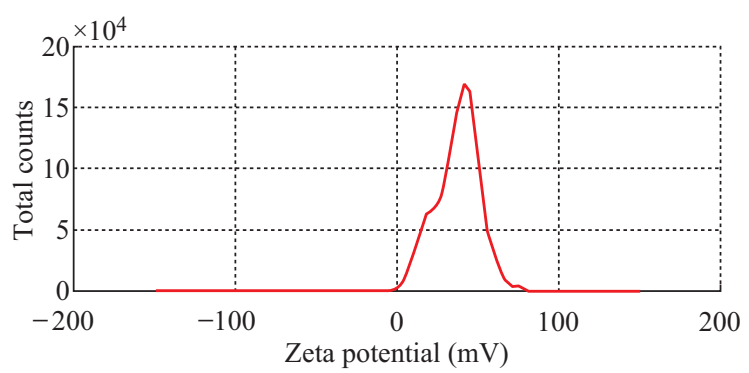

(Run 3)
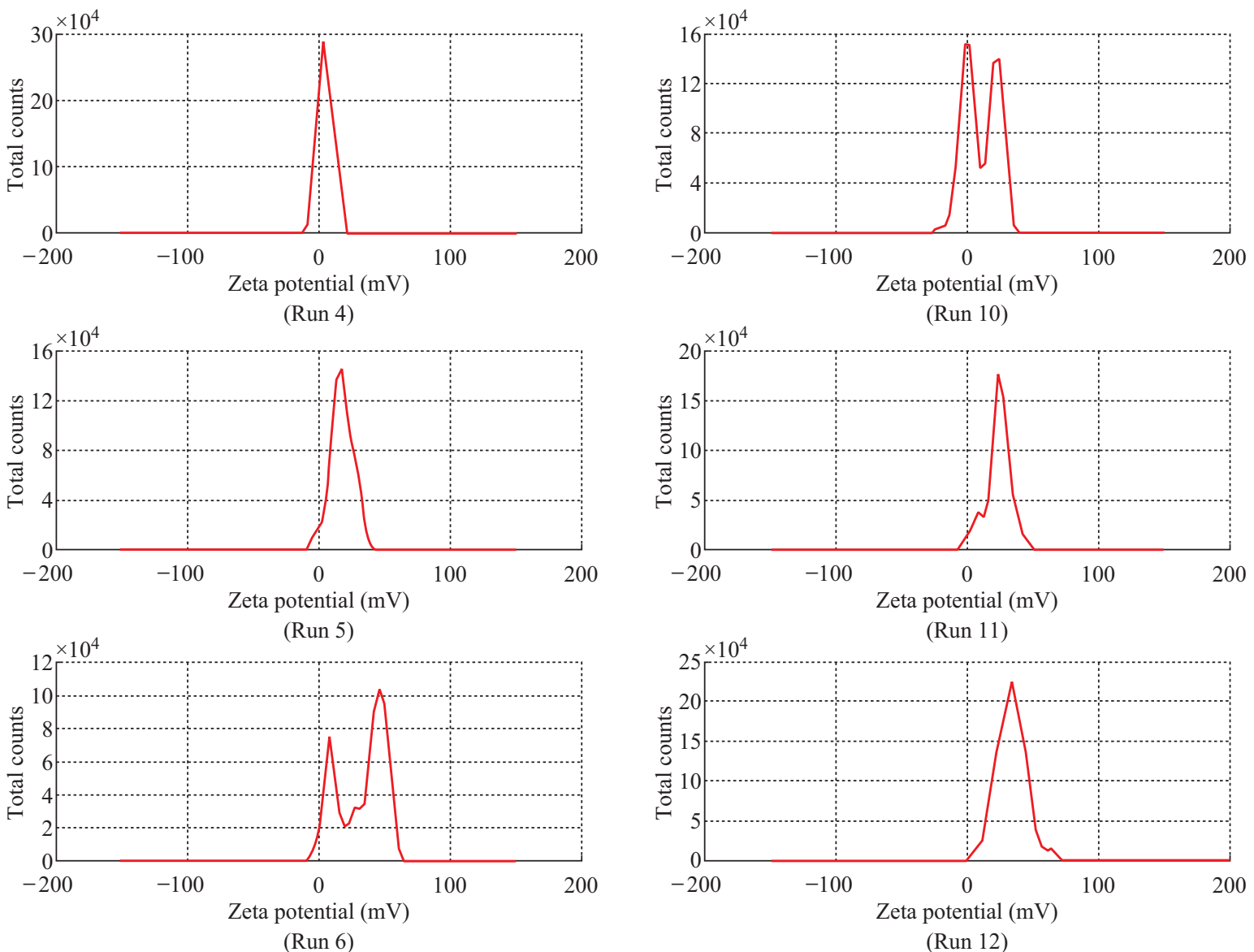

Fig. 2 Zeta potential spectrum of Run 1-12. 
Table 5 ANOVA of average particle size, PDI and zeta potential

\begin{tabular}{|c|c|c|c|c|c|c|}
\hline Variables & Source & Sum of Square & df & Mean of Square & F Ratio & Prob. $>F^{*}$ \\
\hline \multirow{3}{*}{ Average Particle Size } & Model & 115806 & 10 & 11580.60 & & \\
\hline & Residual & 23.21 & 1 & 23.21 & 498.88 & 0.0348 \\
\hline & C. Total & 115829 & 11 & & & \\
\hline \multirow{3}{*}{ Polydispersity Index } & Model & 0.68 & 8 & 0.085 & & \\
\hline & Residual & 0.0043 & 3 & 0.0014 & 58.29 & 0.0033 \\
\hline & C. Total & 0.68 & 11 & & & \\
\hline \multirow{3}{*}{ Zeta Potential } & Model & 817.42 & 2 & 408.71 & & \\
\hline & Residual & 120.32 & 9 & 13.37 & 30.57 & $<0.0001$ \\
\hline & C. Total & 937.73 & 11 & & & \\
\hline
\end{tabular}

*Prob. $>\mathrm{F}$ is the significance level and a value $<0.05$ considered significant

Table 6 Statistical analysis of average particle size, PDI and zeta potential

\begin{tabular}{|c|c|c|c|c|c|c|}
\hline & \multicolumn{2}{|c|}{ Average Particle Size } & \multicolumn{2}{|c|}{ Polydispersity Index } & \multicolumn{2}{|c|}{ Zeta Potential } \\
\hline & Coefficient & P Value & Coefficient & P Value & Coefficient & P Value \\
\hline $\mathrm{b}_{0}$ & 155.09 & 0.0348 & 0.3084 & 0.0033 & 22.53 & $<0.0001$ \\
\hline A & 11.21 & 0.0786 & -0.1194 & 0.0017 & 6.06 & 0.0003 \\
\hline $\mathrm{B}$ & 56.19 & 0.0158 & 0.1008 & 0.0028 & -0.7333 & 0.2337 \\
\hline $\mathrm{C}$ & 35.20 & 0.0251 & 0.1203 & 0.0016 & -1.2667 & 0.1800 \\
\hline $\mathrm{D}$ & -28.74 & 0.0308 & 0.0073 & 0.1000 & -0.3333 & 0.1000 \\
\hline $\mathrm{E}$ & -15.63 & 0.0565 & -0.0338 & 0.0548 & 1.15 & 0.1889 \\
\hline $\mathrm{F}$ & -13.92 & 0.0634 & -0.0311 & 0.0666 & -5.61 & 0.0005 \\
\hline G & 45.77 & 0.0193 & 0.0149 & 0.2179 & -1.54 & 0.1541 \\
\hline $\mathrm{H}$ & -37.50 & 0.0236 & -0.0094 & 0.4177 & 0.89 & 0.2056 \\
\hline $\mathrm{J}$ & 9.13 & 0.0962 & -0.0603 & 0.0120 & 0.5167 & 0.3648 \\
\hline $\mathrm{K}$ & 16.71 & 0.0529 & 0.0868 & 0.0043 & 1.3666 & 0.1765 \\
\hline $\mathrm{L}$ & -1.39 & 0.1000 & 0.0664 & 0.0091 & -1.0733 & 0.1758 \\
\hline
\end{tabular}

Table 7 Observed (O) \& Predicted (P) value of size, PDI and zeta potential

\begin{tabular}{|c|c|c|c|c|c|c|}
\hline \multirow{2}{*}{ Run } & \multicolumn{2}{|c|}{ Average Particle Size } & \multicolumn{2}{|c|}{ Polydispersity Index } & \multicolumn{2}{|c|}{ Zeta Potential } \\
\hline & $\mathbf{O}$ & $\mathbf{P}$ & $\mathbf{O}$ & $\mathbf{P}$ & $\mathbf{O}$ & $\mathbf{P}$ \\
\hline 1 & 159.00 & 160.39 & 0.910 & 0.930 & 20.8 & 22.08 \\
\hline 2 & 125.50 & 126.89 & 0.060 & 0.092 & 23.2 & 22.98 \\
\hline 3 & 102.10 & 100.71 & 0.131 & 0.120 & 19.5 & 22.98 \\
\hline 4 & 290.50 & 291.89 & 0.641 & 0.620 & 4.96 & 10.86 \\
\hline 5 & 66.04 & 64.65 & 0.311 & 0.340 & 17.2 & 10.86 \\
\hline 6 & 426.50 & 425.11 & 0.452 & 0.430 & 31.9 & 34.19 \\
\hline 7 & 129.70 & 128.31 & 0.187 & 0.190 & 26.9 & 22.98 \\
\hline 8 & 136.50 & 135.11 & 0.205 & 0.190 & 23.0 & 22.08 \\
\hline 9 & 116.40 & 117.79 & 0.177 & 0.180 & 36.8 & 34.19 \\
\hline 10 & 133.20 & 134.59 & 0.334 & 0.300 & 9.76 & 10.86 \\
\hline 11 & 78.07 & 76.68 & 0.166 & 0.180 & 23.1 & 22.08 \\
\hline 12 & 97.62 & 99.01 & 0.127 & 0.130 & 33.2 & 34.19 \\
\hline
\end{tabular}

of nanoparticles. PDI value less than 0.5 indicates the narrow sized distribution, which is the most significant parameter that decides the consistency of nanoparticle performance such as solubility, dissolution, drug release, cellular uptake. However, PDI value greater than 0.5 indicates the aggregation of particles or broad sized distribution, which leads to difficulty in establishing the conclusion on which sized particles are responsible for the biological effects. [19, 20]. Hence, it is essential to fabricate polymeric nanoparticles with PDI less than 0.5. Analysis of variance has shown that the process parameters has significant effect (Prob.>
F, 0.0033) on PDI (Table 5). All process parameters except $\beta$-cyclodextrin concentration, aqueous phase volume and organic phase volume significantly influence the polydispersity index (Table 6 and Fig. 4).

Parameters such as organic solvent percentage, organic solvent, stirring speed, stirring duration, $\beta$-cyclodextrin concentration and organic phase volume has favourable effect on the PDI whereas, polymer concentration, beaker volume, poloxamer 188 concentration, poloxamer 407 concentration and aqueous phase volume has inverse relationship with the PDI (Fig. 4). Moreover, the observed PDI were 
comparable with predicted value of Plackett-Burman factorial design (Table 7). The linear model explaining the effects of process parameters on PDI is given as PDI $=+0.31-0.12 * \mathrm{~A}+0.10 * \mathrm{~B}+0.12 * \mathrm{C}-0.034 * \mathrm{E}-$ $0.031 * \mathrm{~F}-0.060 * \mathrm{~J}+0.087 * \mathrm{~K}+0.066 * \mathrm{~L}$.

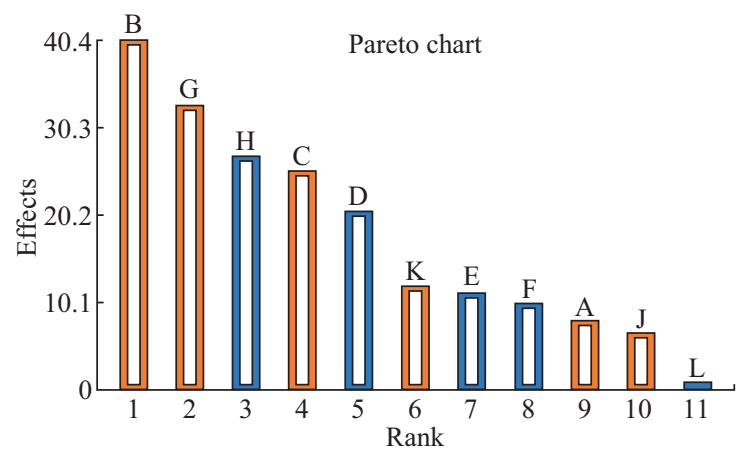

Fig. 3 Plackett-Burman Pareto-Plot for the average particle size using eleven process parameters. Blue colour column indicates the parameter has negative effect and orange colour indicates positive effect on the average particle size. The white column inside both blue and orange columns indicates that the parameter has significant effect on average particle size.

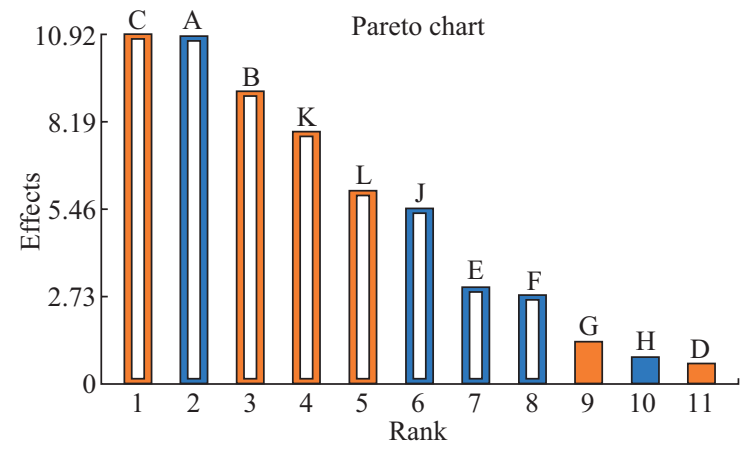

Fig. 4 Plackett-Burman Pareto-Plot for polydispersity index using eleven process parameters. Blue colour column indicates the parameter has negative effect and orange colour indicates positive effect on the PDI. The white column inside both blue and orange columns indicates that the parameter has significant effect on PDI.

\section{Effect of process parameters on the zeta potential}

The stability of nanoformulation depends on the charge around the nanoparticles. Higher number of likely charged nanoparticles repels each other and creates an electrostatic repulsive force and maintains the nanoparticles in Brownian motion, which is expected to overcome the van der walls attractive force arising from induced dipole-dipole interaction between nanoparticles and gravitational force, thereby stabilize the nanoformulation by preventing the agglomeration. Nanoparticles with zeta potentials $< \pm 20 \mathrm{mV}$ are have limited stability $[20,21]$. Analysis of variance has shown that the process parameters has significant effect (Prob> F, <0.0001) on zeta potential (Table 5). Except polymer concentration and poloxamer 407 concentration, all other process parameters do not significantly influence the zeta potential (Table 6 and Fig. 5).

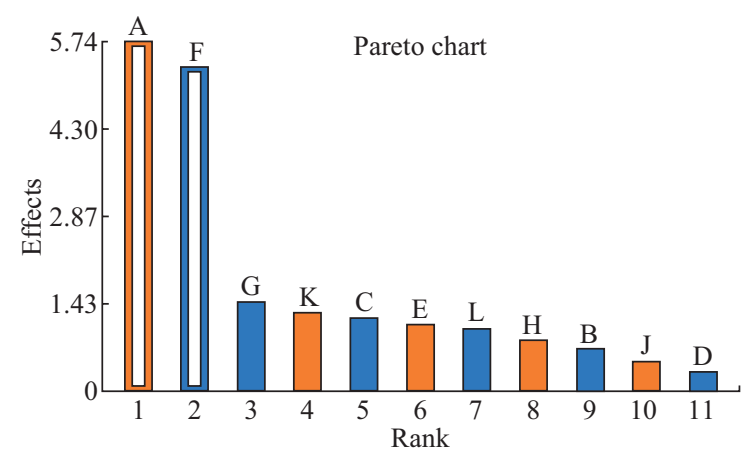

Fig. 5 Plackett-Burman Pareto-Plot for zeta potential using eleven process parameters. Blue colour column indicates the parameter has negative effect and orange colour indicates positive effect on the zeta potential. The white column inside both blue and orange columns indicates that the parameter has significant effect on zeta potential.

Parameters such as polymer concentration, stirring speed, poloxamer 188 concentration, aqueous phase volume and beaker volume has favourable effect on the zeta potential whereas, poloxamer 407 concentration, $\beta$-cyclodextrin concentration, organic solvent percentage, stirring duration, organic solvent and organic phase volume has inverse relationship with the zeta potential (Fig. 5). Moreover, the observed zeta potential values were comparable with predicted value (Table 7) except $4^{\text {th }}$ and $5^{\text {th }}$ run. The linear model explaining the effects of process parameters on zeta potential is given as Zeta potential $=+22.53+6.06 * \mathrm{~A}$ $-5.61 * \mathrm{~F}$.

The optimized formula (with desirability: 0.97) for the fabrication of dimethylaminoethyl methacrylate based nanoparticulate drug delivery system was displayed in RAMPS format (Fig. 6). However, values were rounded-off and used in the fabrication of plain and curcumin-piperine, curcumin-quercetin and curcumin-silibinin loaded polymeric nanoparticles.

\section{Fabrication of plain, curcumin-piperine, curcumin-quercetin and curcumin- silibinin loaded dimethylaminoethyl methacrylate based nanoparticles}

Plain, curcumin-piperine, curcumin-quercetin and curcumin-silibinin loaded dimethylaminoethyl 

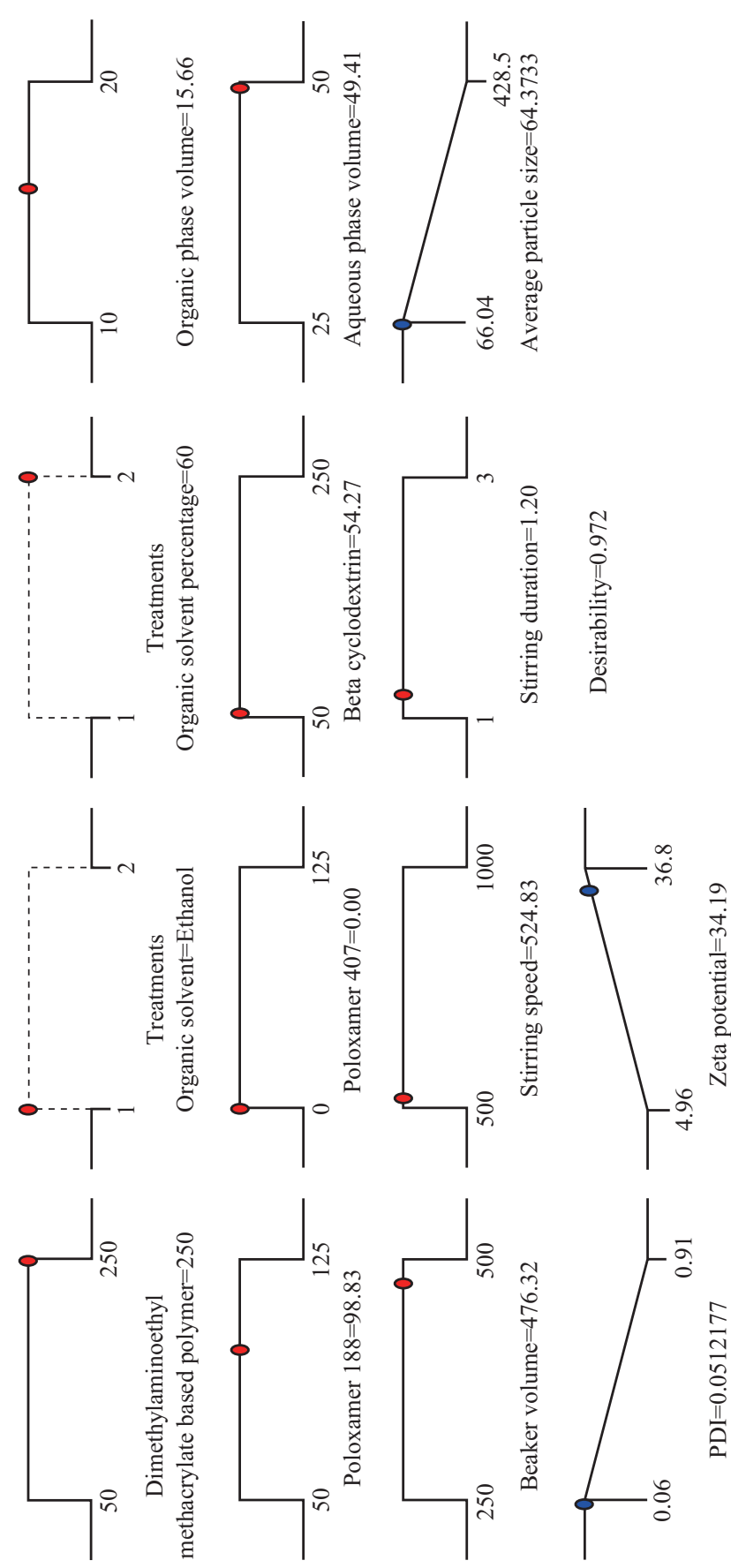

Fig. 6 RAMPS format of optimized formula for the fabrication of dimethylaminoethyl methacrylate based nanoparticulate drug delivery system.

methacrylate based nanoparticles were prepared using final optimized formula from Plackett-Burman factorial design. About $250 \mathrm{mg}$ of dimethylaminoethyl methacrylate polymer with and without $50 \mathrm{mg}$ of various dual drug combinations (Curcumin $25 \mathrm{mg}+$ Piperine $25 \mathrm{mg}$ ); (Curcumin $25 \mathrm{mg}+$ Quercetin 25 $\mathrm{mg}$ ); and (Curcumin $25 \mathrm{mg}+$ Silibinin $25 \mathrm{mg}$ ) were dissolved in $16 \mathrm{~mL}$ of $60 \%$ ethanol. The prepared organic phase was transferred at once into $500 \mathrm{~mL}$ beaker containing $49 \mathrm{~mL}$ of distilled water, $97 \mathrm{mg}$ of Poloxamer 188 and $54 \mathrm{mg}$ of $\beta$-cyclodextrin under mechanical stirring (RQT-124A, Remi) at $520 \mathrm{rpm}$. Polymeric nanoparticles were formed spontaneously but the stirring process was continued for 80 minutes to aid the size reduction and to evaporate the residual solvent. The fabrication experiments were performed in triplicate. After fabrication, prepared plain, curcumin-piperine, curcumin-quercetin and curcuminsilibinin loaded nanoformulations were stored at room temperature for one month to identify any aggregation and post-formulation degradation. Prepared plain, curcumin-piperine, curcumin-quercetin and curcuminsilibinin loaded polymeric nanoparticles were characterized for particle size, polydispersity index and zeta potential. The characterization results were summarized in Table 8 and characterization spectrums were displayed in Fig. 7.

After fabrication, prepared plain, curcuminpiperine, curcumin-quercetin and curcumin-silibinin loaded polymeric nanoformulations were stored at room temperature for one month. However, there was no aggregation. Plain polymeric nanoparticles have shown an average particle size of $64.42 \mathrm{~nm}$ with PDI of 0.234 and zeta potential of $29.3 \mathrm{mV}$. Encapsulation of curcumin-piperine and curcumin-quercetin has increased the average particle size $(86.87 \mathrm{~nm}$ and $71.03 \mathrm{~nm})$, increased the PDI (0.239 and 0.281) and decreased the zeta potential $(22.2 \mathrm{mV}$ and 38.8 $\mathrm{mV})$. However, encapsulation of curcumin-silibinin has decreased the average particle size $(46.95 \mathrm{~nm})$, decreased the zeta potential $(26.6 \mathrm{mV})$ and decreased the PDI (0.142).

We hypothesis the following (a) After encapsulation

Table 8 Average particles size, polydispersity index and zeta potential of prepared plain and dual drug loaded polymeric nanoparticles

\begin{tabular}{cccc}
\hline Dual Drug Combinations & Average Particle Size (nm) & Polydispersity Index & Zeta Potential (mV) \\
\hline Plain & $64.42 \pm 0.920$ & $0.234 \pm 0.250$ & $29.3 \pm 1.16$ \\
Curcumin-Piperine & $86.87 \pm 0.760$ & $0.239 \pm 0.140$ & $22.2 \pm 1.50$ \\
Curcumin-Quercetin & $71.03 \pm 0.741$ & $0.281 \pm 0.110$ & $38.8 \pm 7.70$ \\
Curcumin-Silibinin & $46.95 \pm 0.71$ & $0.142 \pm 0.170$ & $26.6 \pm 4.40$ \\
\hline
\end{tabular}




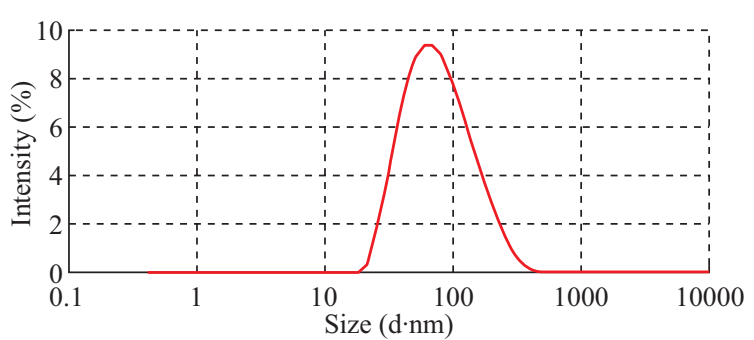

(A)

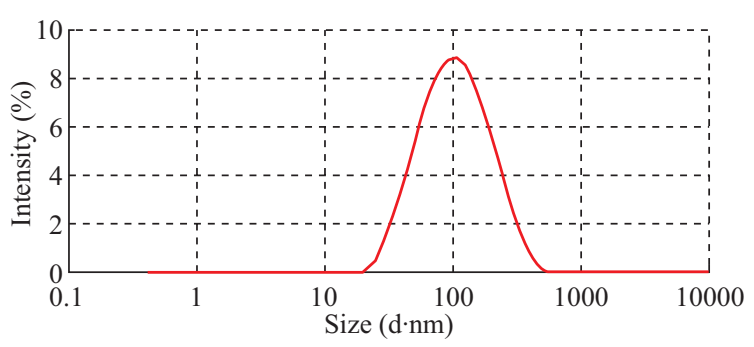

(B)

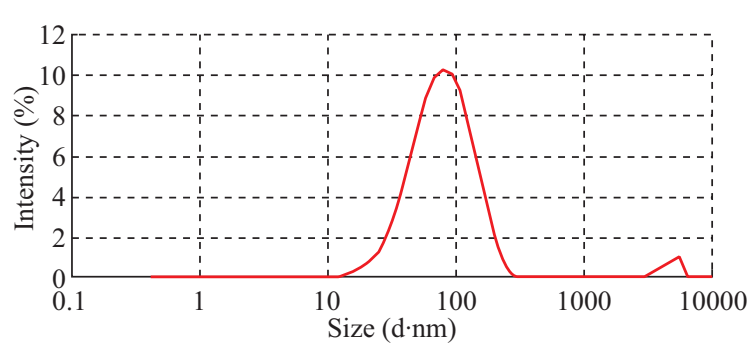

(C)

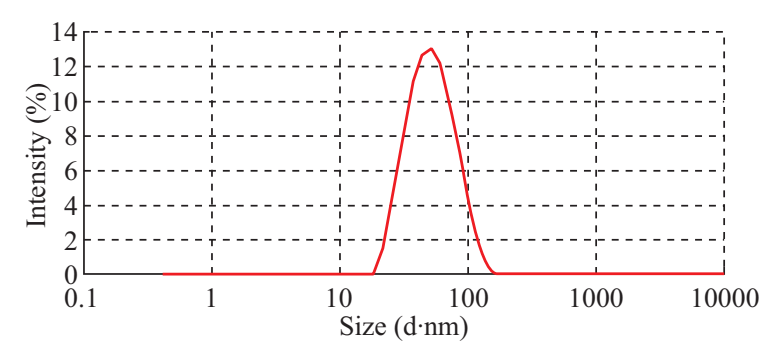

(D)

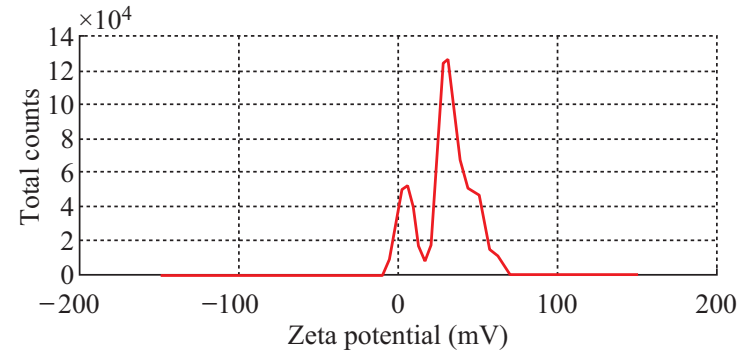

(A1)

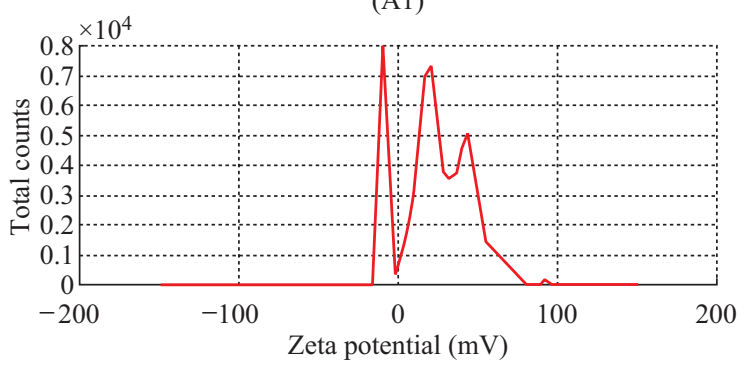

(B1)

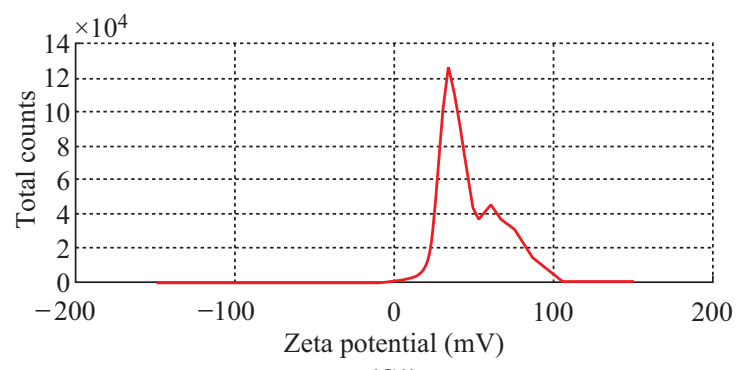

(C1)

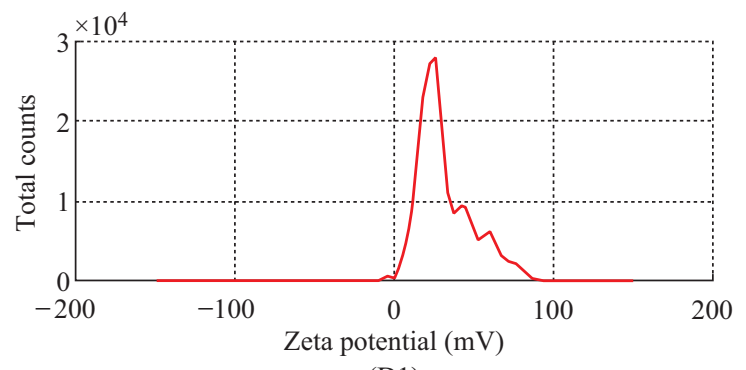

(D1)

Fig. 7 Particle size spectrum of plain (A), Curcumin-Piperine (B), Curcumin-Quercetin (C), Curcumin-Silibinin (D) and Zeta potential spectrum of dummy (A1), Curcumin-Piperine (B1), Curcumin-Quercetin (C1), and Curcumin-Silibinin (D1).

of curcumin (Fig. 8) and piperine (Fig. 9), average particle size increased due to accommodation of these drugs in polymeric nanoparticles. Moreover, both curcumin and piperine are cationic in nature and expected to repel each inside the nanoparticles, which might have increased the size. Similarly, increase in particle size might have increased the PDI. (b) After encapsulation of curcumin (Fig. 8) and quercetin (Fig. 10), average particle size increased due to accommodation of these drugs in polymeric nanoparticles. Moreover, curcumin possess strong cationic nature and quercetin possess week anionic nature, which are expected to attract each other inside the nanoparticles and increase in particle size due to repel force has not occurred in this formulation. Hence, curcumin-quercetin combination has produced lesser particle size than the curcumin-piperine combination. Similarly, increase in particle size might have increased the PDI. (c) After encapsulation of curcumin (Fig. 8) and silibinin (Fig. 11), average particle size reduced significantly. The reason might be curcumin possess strong cationic nature and silibinin possess strong anionic nature, which are expected to attract each other strongly inside the nanoparticles, which might have caused significant size reduction than curcuminquercetin \& curcumin-piperine combination. Similarly, significant decrease in particle size might have decreased the PDI significantly. (d) Encapsulation of 
<smiles>COc1cc(/C=C/C(=O)CC(=O)/C=C/c2ccc(O)c(OC)c2)ccc1O</smiles>

Fig. 8 Chemical structure of curcumin.

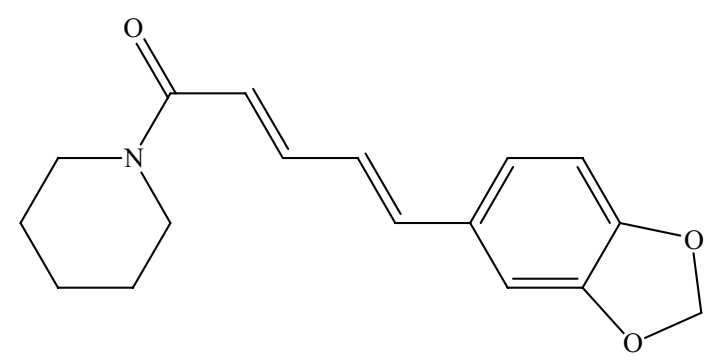

Fig. 9 Chemical structure of piperine.

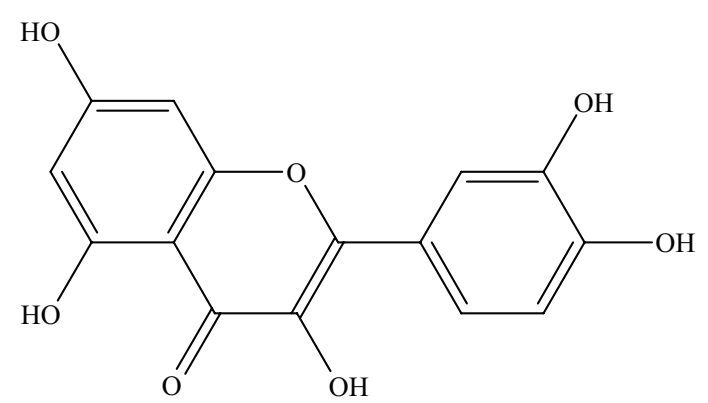

Fig. 10 Chemical structure of quercetin.<smiles>COc1cc([C@H]2Oc3cc([C@H]4Oc5cc(O)cc(O)c5C(=O)[C@H]4O)ccc3O[C@@H]2CO)ccc1O</smiles>

Fig. 11 Chemical structure of silibinin.

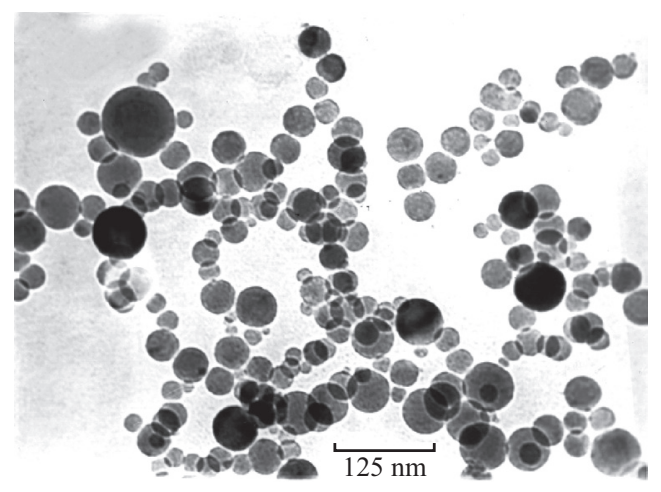

Fig. 12 TEM image of Curcumin-Quercetin dimethylaminoethyl methacrylate based nanoparticles. curcumin-piperine, curcumin-quercetin and curcuminsilibinin showed significant change in zeta potential but the rationale is not clear.

Particle surface morphology decides the basic function of particles, degradation, release of drug from polymer matrix, transport of particles in the body, internalization of drug. Prepared plain, curcuminpiperine, curcumin-quercetin and curcumin-silibinin loaded polymeric nanoparticles were imaged using transmission electron microscope and found to be spherical in shape (Fig. 12). Hence, curcumin and bioenhancer piperine, quercetin and silibinin encapsulated in the polymer matrix will also be in spherical shape and expected to enhance the basic function of curcumin and bio-enhancers, release of curcumin and bio-enhancers from the polymer matrix, transport of curcumin and bio-enhancers in the body and internalization of curcumin and bio-enhancers by many folds than the free curcumin and bio-enhancers.

\section{Conclusion}

In the present study, dimethylaminoethyl methacrylate based nanoparticulate drug delivery system was developed using nanoprecipitation method. Effect of eleven process parameters on average particle, polydispersity index and zeta potential were studied using Plackett-Burman factorial design. Average particles size $<100 \mathrm{~nm}$, polydispersity index $<0.5$ and zeta potential $> \pm 20 \mathrm{mV}$ was used to evaluate the quality of the prepared nanoparticles. The optimized formula was $250 \mathrm{mg}$ of dimethylaminoethyl methacrylate based polymer, $50 \mathrm{mg}$ of various dual drug combinations, $16 \mathrm{~mL}$ of $60 \%$ of ethanol, $500 \mathrm{~mL}$ beaker, $49 \mathrm{~mL}$ of distilled water, $97 \mathrm{mg}$ of poloxamer $188,54 \mathrm{mg}$ of $\beta$-cyclodextrin, stirring speed was $520 \mathrm{rpm}$ and stirring duration was 80 minutes. The optimized formula was implemented to fabricate curcumin-piperine, curcumin-quercetin and curcumin-silibinin loaded dimethylaminoethyl methacrylate nanoparticles and the prepared nanoparticles were well within acceptable limits. The developed dimethylaminoethyl methacrylate based nanoparticulate drug delivery system was simple and can be routinely used to fabricate narrow sized polymeric nanoparticles without filtration or centrifugation process.

\section{Acknowledgments}

C. Moorthi is thankful to UGC, Government of 
India, for providing UGC-BSR fellowship.

\section{References}

[1] Moorthi C., Kathiresan K., Nanotoxicology: Toxicity of engineered nanoparticles and approaches to produce safer nanotherapeutics. Int. J. Pharma. Sci. 2012; 2: 117-124.

[2] Moorthi C., Manavalan R., Kathiresan K., Nanotherapeutics to overcome conventional cancer chemotherapy limitations. J. Pharm. Pharm. Sci. 2011; 14: 67-77.

[3] Kesisoglou F., Panmai S., Wu Y., Nanosizing - oral formulation development and biopharmaceutical evaluation. Adv. Drug Deliver. Rev. 2007; 59: 631-644.

[4] Moorthi C., Kathiresan K., Curcumin-Piperine/ Curcumin-Quercetin/Curcumin-Silibinin dual drugloaded nanoparticulate combination therapy: A novel approach to target and treat multidrug-resistant cancers. $J$. Med. Hypotheses Ideas. 2013; 7: 15-20.

[5] Gelperina S., Kisich K., Iseman M.D., Heifets L., The potential advantages of nanoparticle drug delivery systems in chemotherapy of tuberculosis. Am. J. Resp. Crit. Care. 2005; 172: 1487-1490.

[6] Moorthi C., Kathiresan K., Application of PlackettBurman factorial design in the development of curcumin loaded Eudragit E 100 nanoparticles. Nano Biomed. Eng. 2013; 5: 28-33.

[7] De Jong W.H., Borm P.J., Drug delivery and nanoparticles: Applications and hazards. Int. J. Nanomed. 2008; 3: 133-149.

[8] Moorthi C., Kathiresan K., Fabrication of dual drug loaded polymeric nanosuspension: Incorporating analytical hierarchy process and data envelopment analysis in the selection of a suitable method. Int. J. Pharm. Pharm. Sci. 2013; 5: 499-504.

[9] Moorthi C., Kathiresan K., A step-by-step optimization process to fabricate narrow sized dual drug loaded polymeric nanoparticles using modified nanoprecipitation technique. Nano Biomed. Eng. 2013; 5(3): 107-115.

[10] Moorthi C., Kiran K., Manavalan R., Kathiresan K., Preparation and characterization of curcumin-piperine dual drug loaded nanoparticles. Asian Pac. J. Trop. Biomed. 2012; 2(11): 841-848.

[11] Moorthi C., Senthil kumar C., Mohan S., Kiran K., Kathiresan K., Application of validated RP-HPLC-PDA method for the simultaneous estimation of curcumin and piperine in Eudragit E 100 nanoparticles. Journal of
Pharmacy Research. 2013; 7: 224-229.

[12] Moorthi C., Senthil kumar C., Mohan S., Kathiresan K., SLS/ $\beta C D$-curcumin nanosuspension: Preparation, characterization and pharmacological evaluation. Journal of Pharmacy Research. 2013; 7: 219-223.

[13] Moorthi C., Kathiresan K., Reversed phase high performance liquid chromatographic method for simultaneous estimation of curcumin and quercetin in pharmaceutical nanoformulation. Int. J. Pharm. Pharm. Sci. 2013; 5(3): 622-625.

[14] Moorthi C., Kathiresan K., Simultaneous estimation of curcumin and silibinin using validated RP-HPLCPDA method and its application in pharmaceutical nanoformulation. Int. J. Pharm. Pharm. Sci. 2013; 5(3): 475-478.

[15] Mora-Huertas C.E., Fessi H., Elaissari A., Polymer-based nanocapsules for drug delivery. Int. J. Pharm. 2010; 385(1-2): 113-142.

[16] Barichello J.M., Morishita M., Takayama K., Nagai T., Encapsulation of hydrophilic and lipophilic drugs in PLGA nanoparticles by the nanoprecipitation method. Drug Dev. Ind. Pharm. 1999; 25(4): 471-476.

[17] Moorthi C., Kathiresan K., Anionic Surfactant Based Topical Curcumin Nanosuspension: Fabrication, Characterization and Evaluation. Nano Biomed. Eng. 2013; 5(2): 86-89.

[18] Moorthi C., Kathiresan K., Fabrication of highly stable sonication assisted curcumin nanocrystals by nanoprecipitation method. Drug Invention Today. 2013; 5: 66-69.

[19] Agarwal V., Bajpai M., Stability issues related to nanosuspensions: A review. Pharmaceutical Nanotechnology. 2013; 1: 85-92.

[20] Bathool A., Vishakante G.D., Khan M.S., Shivakumar H.G., Development and characterization of atorvastatin calcium loaded chitosan nanoparticles for sustain drug delivery. Adv. Mat. Lett. 2012; 3(6): 466-470.

[21] Yassin A.E.B., Anwer M.K., Mowafy H.A., El-Bagory I.M., Bayomi M.A., Alsarra I.A., Optimization of 5-fluorouracil solid-lipid nanoparticles: a preliminary study to treat colon cancer. Int. J. Med. Sci. 2010; 7(6): 398-408.

Copyright $\subset 2014$ Moorthi Chidambaram and Kathiresan Krishnasamy. This is an open-access article distributed under the terms of the Creative Commons Attribution License, which permits unrestricted use, distribution, and reproduction in any medium, provided the original author and source are credited. 